\title{
Sentiment analysis under temporal shift
}

\author{
Jan Lukes and Anders Søgaard \\ Dpt. of Computer Science \\ University of Copenhagen \\ Copenhagen, Denmark \\ smx262@alumni.ku.dk
}

\begin{abstract}
Sentiment analysis models often rely on training data that is several years old. In this paper, we show that lexical features change polarity over time, leading to degrading performance. This effect is particularly strong in sparse models relying only on highly predictive features. Using predictive feature selection, we are able to significantly improve the accuracy of such models over time.
\end{abstract}

\section{Introduction}

Sentiment analysis models often rely on data that is several years old. Such data, e.g., product reviews, continuously undergo shifts, leading to changes in term frequency, for example. We also observe the emergence of novel expressions, as well as the amelioration and pejoration of words (Cook and Stevenson, 2010). Such changes are typically studied over decades or centuries; however, we hypothesize that change is continuous, and small changes can be detected over shorter time spans (years), and that their cumulation can influence the quality of sentiment analysis models. In this paper, we analyze temporal polarity changes of individual features using product reviews data. Additionally, we show that predictive feature selection, trying to counteract shifts in polarity, significantly improves model accuracy over time.

Contributions First, we show deterioration of sentiment analysis model performance over time. We propose rank-based metrics for detecting polarity shifts and identify several examples of lexical features that exhibit temporal drift in our data. Finally, we use our findings to design a predictive feature selection scheme, based on expected polarity changes, and show that models using predictive

\section{Sample positive review:}

"Grand daughters really like this movie. Good clean movie for all ages. Would recommend for everyone. Good horse movie for girls."

\section{Sample negative review:}

"Not what I expected. Very cheap and chintzy looking for the price. Certainly did not look like a wallet. Very disappointed in the quality."

Figure 1: Examples of reviews

feature selection perform significantly better than models that simply rely on the most predictive features across the training data without estimating temporal shifts.

\begin{tabular}{ccccc}
\hline Training data & \multicolumn{4}{c}{ Model accuracy } \\
\hline Runs & 1 & 2 & 3 & Mean \\
\hline $2001-2004$ & 0.858 & 0.855 & 0.863 & 0.859 \\
$2008-2011$ & 0.877 & 0.873 & 0.878 & 0.877 \\
\hline
\end{tabular}

Table 1: Classifier accuracy deterioration when using older and newer training sets. Models were trained using random independent data subsets from periods 2001-2004 or 2008-2011 and tested using random independent subsets from 20122014. Subset size: $K=40,000$ with $80 / 20$ split.

\section{Sentiment analysis models get worse over time}

One way we may observe polarity shifts over time is when we see models trained on older data perform worse than models trained on more recent data. Or, equivalently, by seeing performance degradations over time.

The Amazon product review data, which we 
will use in our experiments, is a collection of user product reviews and meta-data crawled from Amazon.com, consisting of 82 million reviews and spanning May 1996 until July 2014 (He and McAuley, 2016; McAuley et al., 2015). Previous work already preprocessed the dataset, removing duplicates and boiler plates. We sample large subsamples of reviews per year from the original data set $(K=40000)$. Only years 2001 to 2014 were used in this project, as the samples from earlier years were of insufficient size.

Following previous work (Blitzer et al., 2007), we map the user-provided sentiment annotations, ranging from 1 star to 5 stars, into binary labels, where 3 stars and less are replaced by negative class labels, indicating negative or critical review, and 4 or more stars were considered positive reviews and associated with a positive class label.

Experiments with off-the-shelf classifiers In our first set of experiments, we train logistic regression models on data from 2001-2004 and on data from 2008-2011, and test their accuracy on random test samples from years 2012 to 2014 . Our results show that models trained on older data performed noticeably worse than models trained on data from years 2008-2011 (see Table 1). The mean accuracies, obtained by averaging accuracies of models trained on three independently selected samples, were 0.859 for models trained on reviews from 2001-2004, and 0.877 for models trained on reviews from 2008-2011, i.e., an average absolute $2 \%$ decrease in accuracy.

This model deterioration over time could be attributed to a decrease in vocabulary overlap - as measured by, for example, the Jaccard similarity coefficient over unigrams and bigrams. To measure possible influence of this factor, we monitor the Jaccard index of unigram and bigram features that occur at least 5 times in the data sets: The average Jaccard indices between training and test data were 0.112 for 2001-2004 and 0.154 for 2008-2011. ${ }^{1}$ Since the difference is minor, we hypothesize that temporal shifts in polarity are responsible for at least some of the drops in model performance over time. We confirm this hypothesis below by monitoring performance over time with a fixed feature set (fixing also the Jaccard index).

\footnotetext{
${ }^{1}$ The relatively low values are due to training and test set being of different sizes, 32000 and 8000 respectively.
}

\subsection{Experiments with a fixed feature set}

The purpose of our second set of experiments is again to see how accuracy changesover time with models being trained on 'older' and 'newer' data subsets, but on identical feature sets. Similar to the above experiments, years 2001 to 2004 were selected as our older training data subsets, and years 2008 to 2011 were selected as our newer data. We again sample 40,000 reviews per year and create 80/20 train-test splits. The experiment was repeated three times with new samples to obtain the average accuracies seen below in Figure 2 . The fixed feature set used was obtained by selecting the 5,000 most frequent unigrams and bigrams present in the training data for year 2001. We use simple count vectors to represent the reviews.

The deterioration of performance over time is clearly visible from the plot in Figure 2, by looking at the gap between the red and the green scatter points. We believe these results support our hypothesis that over time, the polarities of individual features may change, and the cumulation of such changes significantly influences performance of sentiment classifiers.

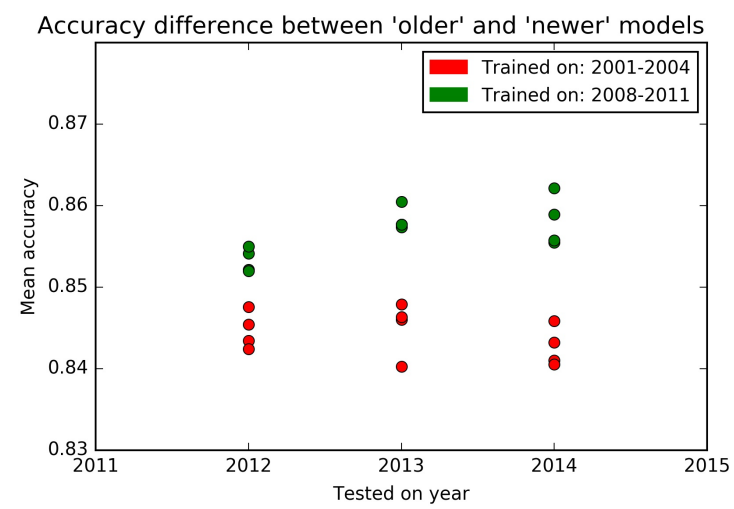

Figure 2: Each dot represents the average accuracy of the model trained on data from year $x$, where $y \in[2001, . ., 2004,2008, . ., 2011]$, and tested on year $x$, where $x \in[2012,2013,2014]$. All possible combinations were run 3 times with different random yearly subsets to compute the average accuracies presented in the figure.

\section{Polarity shifts}

In this section, we look at individual features in order to detect examples of polarity shifts, i.e., amelioration or pejoration over time. We do so by analyzing the weights of classifiers trained on different years. As in our previous experiments, we use 
logistic regression classifiers trained with $\ell_{1}$ regularization penalties. For each year in the interval 2001 to 2014, we training a classifier on a training set of 32,000 randomly sampled reviews, and we then inspect the coefficients associated with particular lexical features. These values measure the impact of lexical features on the final predictions of the models; high coefficients associate lexical features with positive sentiment, and low (negative) coefficients associate lexical features with high negative sentiment.

Logistic regression coefficients are not comparable across models, though, because of different scales, and one option would therefore be to use Min-Max scaling to transform coefficient values to the interval $[1,0]$ for positive values and $[0,-1]$ for negative values. We use such scaling later in the paper; however, for robustly detecting polarity shifts, we instead propose using feature polarity rankings. Such ranking is done by ordering features by their respective coefficients and assigning a rank to each feature. The highest coefficient is rank 1 , the second highest rank 2 , and so on. This allows us to make direct comparisons between several models trained on different subsets of data.

\begin{tabular}{cccc}
\hline Year & \multicolumn{3}{c}{ Positive feature polarity rank } \\
\hline & 'highly' & 'great' & 'incredible' \\
2001 & 1 & 11 & 9 \\
2002 & 4 & 11 & 10 \\
2003 & 4 & 6 & 57 \\
2004 & 1 & 6 & 40 \\
2005 & 3 & 4 & 50 \\
2006 & 3 & 1 & 173 \\
2007 & 6 & 1 & 137 \\
2008 & 3 & 2 & 126 \\
\hline
\end{tabular}

Table 2: Example feature ranks obtained by training a logistic regression classifier on 32,000 reviews from each year.

Due to the exponential distribution of coefficient values, as seen in Figure 3, a cap on maximal rank is placed such that $\max \{\operatorname{rank}\} \leq 3 * f$, where $f$ is the number of positive (or negative) features. If ranks would be uncapped, even the slightest decrease in coefficient value would disproportionately increase feature ranks. As a result, rank and rank averages (used in predictive feature selection) would be much more influenced by randomness of logistic regression, and hence, less interpretable. We argue that shifts in polarity are more precisely measured this way.

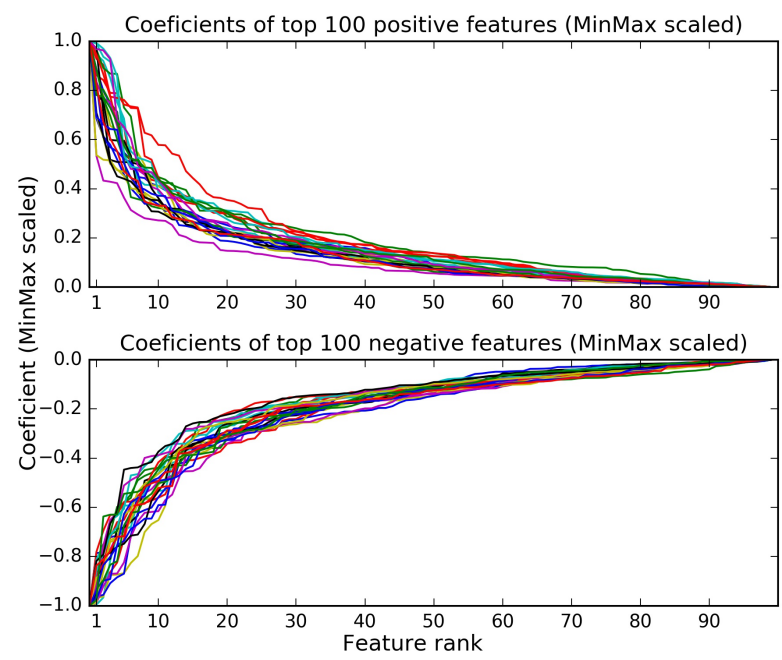

Figure 3: Distribution of coefficient values of 100 most positive and negative features obtained from models trained on data subsets from years 2001 to 2014. Coefficient values were scaled using MinMax.

Using the feature polarity ranking described above, we analyze what shifts occur in individual unigrams and bigrams. To do so, we again use random data subsets of 40,000 reviews and $80 / 20$ splits. For each year spanning 2001 to 2014 , we again generate three independent subsets to allow for more robust results and less randomness caused by logistic regression. Each subset is used to train a classifier, and we compute the polarity ranks of all lexical features.

Once we have established the ranks of lexical features, we use linear regression to estimate the degree to which polarity has changed. Using the $p$ value of such a linear fit, we filter out nonsignificant changes where $p>0.05$. See Figure 4 for examples of significant polarity shifts.

\section{Models that are robust over time}

Based on our previous findings that sentiment analysis models deteriorate over time, as training data sets get older, combined with observing significant changes in the polarity of lexical features, we hypothesize, that predictive feature selection can, to some extent, counteract the negative effects of polarity shift. In our final set of experiments, we perform predictive feature selection using polarity 
Positive feature rank degradation (2001-2014)
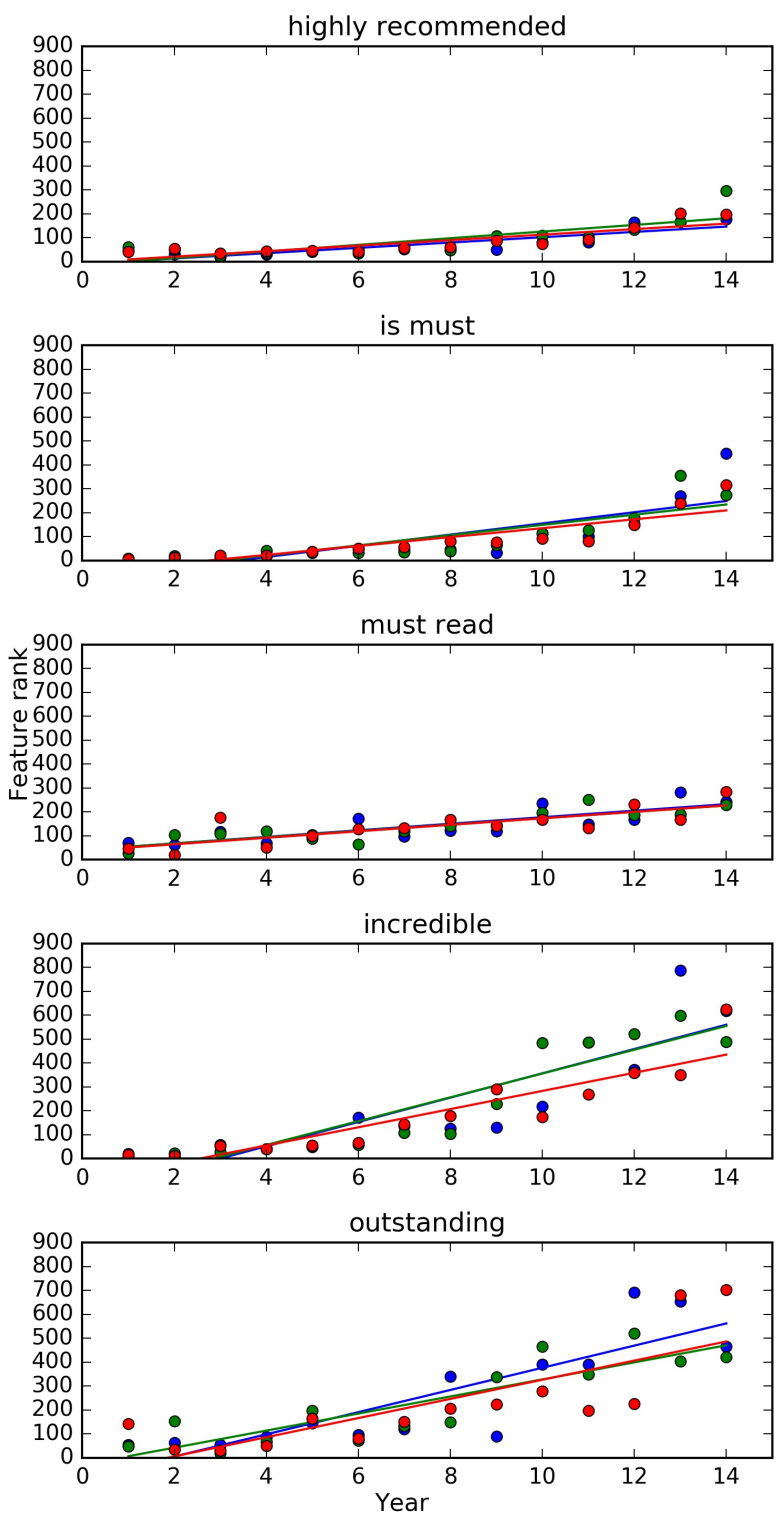

Figure 4: Figure shows a sample of 3 bigrams and 2 unigrams, where significant degradation in positivity was observed. Each color represents an independently selected collection of random yearly subsets of reviews.

rank predictions to select features based on their expected polarity.

We use two methods for predictive feature selection, both relying on the predicted rate (or slope) of change in polarity and a metric of significance that serves as a filtering mechanism. The methods used are:

- Difference of means: This method uses two mean polarity ranks - initial and final, to determine rate of change in feature polarity, and whether the change is significant. Initial and final mean ranks are calculated using polarity ranks from individual years (i.e. how feature ranked in polarity as determined by model trained on data from that year). In our case, the training data spans 2001-2008 and difference of means uses years 2001-2003 for the initial mean rank and 2006-2008 for the final mean rank. Furthermore, each feature to be included has to pass the following significance test:

$$
\left|\frac{\text { mean }(06-08)-\text { mean }(01-03)}{\text { mean }(01-03)}\right|>0.05
$$

The exact value can optionally be determined by experimentation, however in this experiment the significance threshold was set to 5\% of the initial rank.

- Linear regression: We use linear regression to find the trend line of the feature polarity rank and use that in the decision process. As in previous method, yearly polarity ranks are used, however, instead of using only initial or final years we use the whole span of training data to obtain a linear fit. The $p$-value calculated during the fit is used as the significance threshold, where $p<0.05$, for the feature polarity shift to be considered significant.

Using the methods described above, if significant temporal shift in polarity occurs, we obtain an expected rank for each such feature. This information is then used in predictive feature selection that is identical with both methods. First, a feature set of fixed size $K$ (e.g. $K$ positive and $K$ negative features) is created by looking at features that have the best average rank. Additionally, an extended supplement feature set is made of features that are in the next best category (i.e. mean rank $K+1$ to mean rank $3 K$ ). This next best feature set is then analyzed by one of the predictive methods described above, and expected rank is determined for features with significant temporal polarity shift. The results of such analysis are ordered from the most polar expected rank to the least polar, while any result where the expected rank is larger than the fixed size $K$ is ignored. The next step is to analyze the $K$-sized original feature set using the same method. Results are then ordered in inverse order, so that features expected to be least polar are first, while also, any feature that is expected to remain polar (i.e. feature_rank $<K$ ) is ignored. 
This new smaller set of features is then read from least polar expected rank, and while there is a feature in the next best set we make a switch. Simply put, this procedure eliminates features predicted to lose polarity and features predicted to gain most polarity are included instead. Experiments show that number of features switched changes based on parameters (i.e. p-values, size of $K$, etc.) from around $10 \%$ of the feature set for $K=100$ to $30 \%$ for $K=300$.

\subsection{Experiments}

In the first experiment with temporally robust models, we used the difference of means to implement predictive feature selection. The data used to create baseline accuracy was a random subset of $R$ reviews, where, again, $R=40,000$ reviews were selected uniformly at random from years 2001 to 2008. After using $80 / 20$ splits, a subset of 32,000 reviews was used to train a logistic regression classifier, and following the training, $K$ most negative and $K$ most positive features were selected. In contrast to the baseline model, the temporally robust model used the difference of means method to select $K$ negative and $K$ positive features using predictive feature selection described in the section above. This setup was run 3 times with different random subsets of data for both the baseline and the temporally robust model. The result obtained from the runs can be seen below in Figure 5 and Table 3.

\begin{tabular}{ccc}
\hline \multicolumn{3}{c}{ Average model accuracies using 200 features } \\
\hline Test years & Baseline & Temp. robust \\
\hline 2010 & 0.828 & 0.834 \\
2011 & 0.821 & 0.825 \\
2012 & 0.834 & 0.839 \\
2013 & 0.845 & 0.847 \\
2014 & 0.845 & 0.85 \\
\hline
\end{tabular}

Table 3: Each value in the table represents an average accuracy of either a baseline or a temporally robust model tested on a data set of 8,000 reviews from the designated year. Each average is made over 3 experimental runs using different random subsets as training and test data (with no overlap between training and test). Every model used 100 positive and 100 negative features (i.e. $K=100$ ). The predictive feature selection was implemented using the difference of means method.

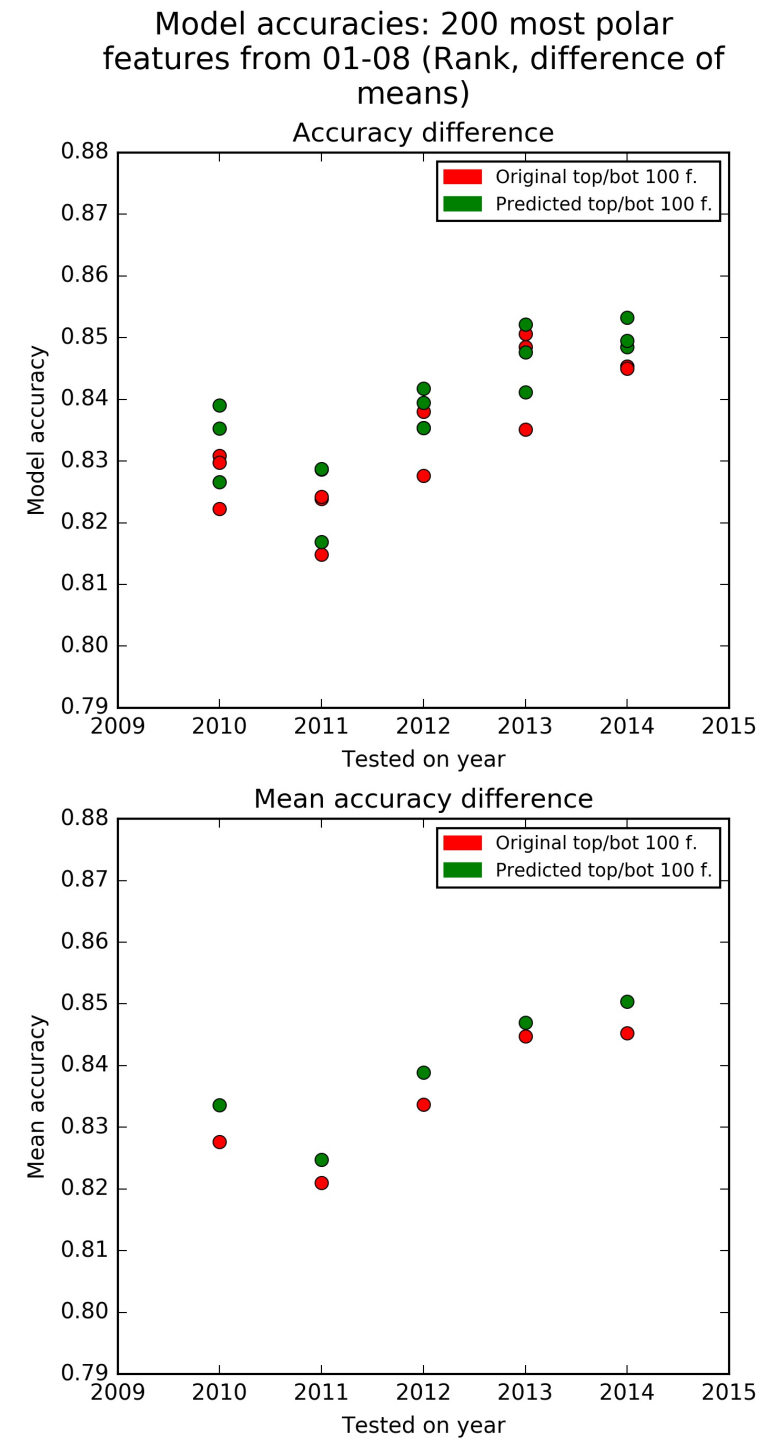

Figure 5: The figure contains results from both the baseline and the temporally robust models trained with 200 features on 32,000 reviews. Upper part of the figure depicts model accuracies for all tested years and all 3 experimental runs; the lower part shows the average accuracy for each tested year. The difference of means method was used for predictive feature selection.

In this particular experiment $K=100$ (i.e. 200 features in total), however, identical experiments were run also with $K=200$ and $K=400$ (see Figure 6). The results indicate that with the number of features limited to 200 , the predictive feature selection on average outperforms our baseline model by a significant margin across all tested years, i.e. 2010 to 2014. 
Model accuracies: 800 most polar features from 01-08 (Rank, difference of means)
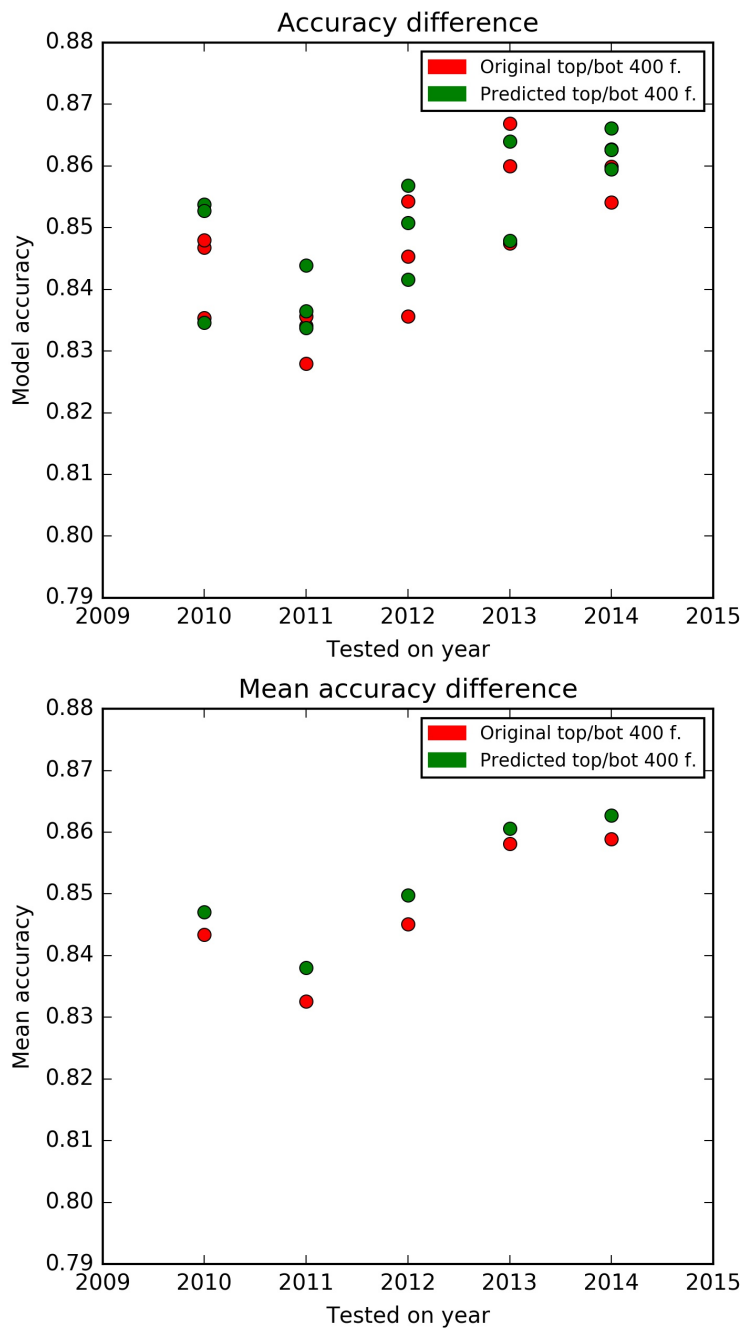

Figure 6: The figure contains results from both the baseline and the temporally robust models trained with 800 features on 32,000 reviews. Upper part of the figure depicts model accuracies for all tested years and all 3 experimental runs; the lower part shows the average accuracy for each tested year. The difference of means method was used for the predictive feature selection.

As can be seen above in Figure 6, significantly increased performance is present even in models that use increased number of features, i.e. from $K=100$ to $K=400$. Such a result suggests that predictive feature selection increases performance even when more features are used, and not only in the extremely sparse model with 200 features.
Model accuracies: 800 most polar features from 01-08 (Rank, pval + linregress)
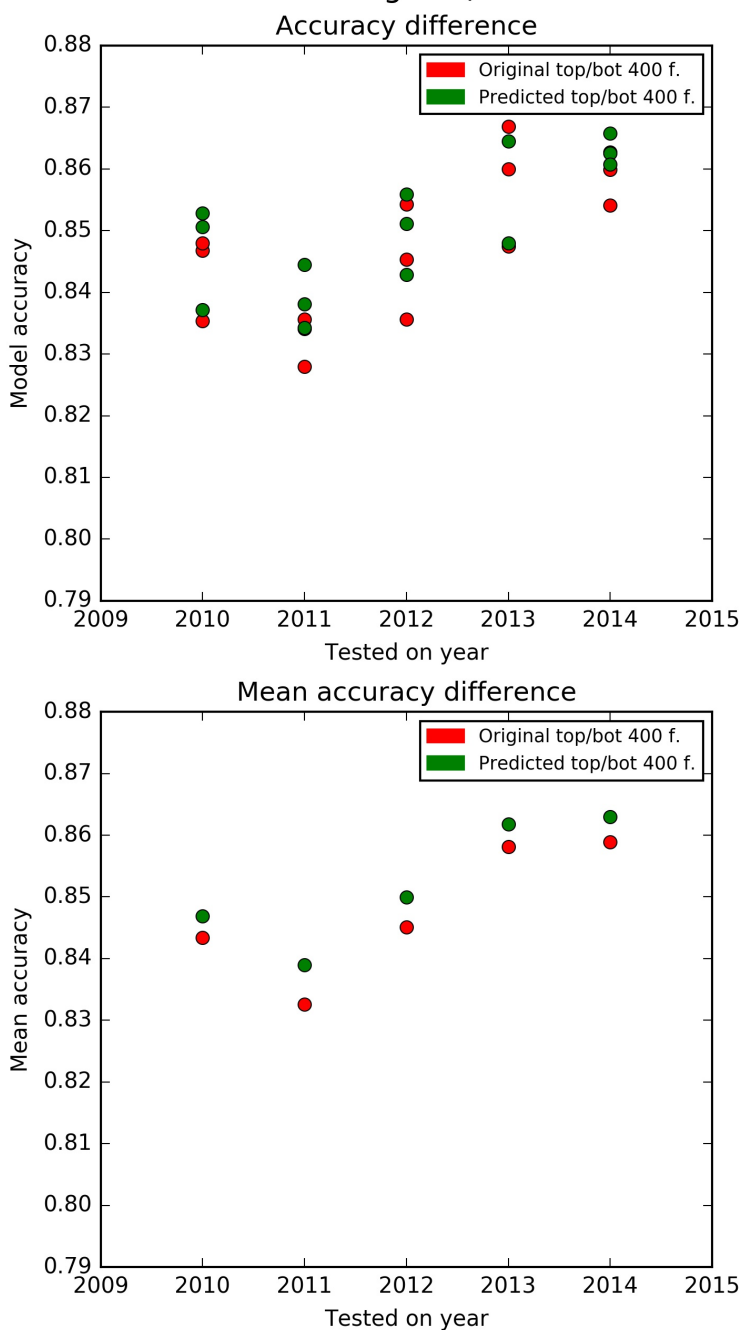

Figure 7: The figure contains results from both the baseline and the temporally robust models trained with 800 features on 32,000 reviews. Upper part of the figure depicts model accuracies for all tested years and all 3 experimental runs; the lower part shows the average accuracy for each tested year. The linear regression method with $p$-value filtering was used for the predictive feature selection.

Furthermore, additional experiment with an identical experimental setup $(K=400)$ was performed; however, the predictive feature selection was implemented using linear regression method with $p$-value filter, as described above in the paper. Results, seen in Figure 7, clearly show that this method also performs better than the baseline approach consisting of selecting only most polar 
features based on training data. In general, both methods - difference of means and linear regression with $p$-value filter - achieve a similar performance, which is consistently better than our baseline model for all tested years and all tested numbers of features, i.e. $K \in[100,200,400]$.

\section{Conclusion}

Large data sets for sentiment analysis are costly to create and are quite commonly a few years old. The performance of classifiers trained on such data sets decreases over time, as the interval between creations of test and training sets expands. This is, in part, due to a cumulative effect of individual lexical features going through amelioration, or pejoration, which significantly changes their polarity over time. We call this effect for temporal polarity shift.

To counter effects of these shifts, and improve the overall performance of a classifier, we devised two methods that allow us to predict the expected feature polarity. Using these methods, we implemented predictive feature selection, an approach, especially beneficial for sparse models, that selects a better feature set for the classifier using the expected polarity, rather than using the current most polar features in the training data. Temporally robust models, i.e., the models using predictive feature selection, consistently achieve better accuracy than the baseline models, which suggest that negative effects of temporal polarity shifts can be countered to some degree.

\section{References}

John Blitzer, Mark Dredze, and Fernando Pereira. 2007. Biographies, Bollywood, Boom-boxes and Blenders: Domain Adaptation for Sentiment Classification. In Proceedings of ACL.

Paul Cook and Suzanne Stevenson. 2010. Automatically identifying changes in the semantic orientation of words. In LREC.

Ruining He and Julian McAuley. 2016. Ups and downs: Modeling the visual evolution of fashion trends with one-class collaborative filtering. In proceedings of the 25th international conference on world wide web, pages 507-517. International World Wide Web Conferences Steering Committee.

Julian McAuley, Christopher Targett, Qinfeng Shi, and Anton Van Den Hengel. 2015. Image-based recommendations on styles and substitutes. In Proceedings of the 38th International ACM SIGIR Confer- ence on Research and Development in Information

Retrieval, pages 43-52. ACM. 